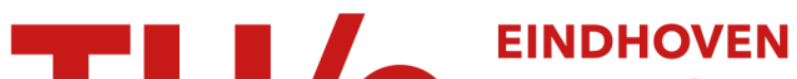 \\ UNIVERSITY OF \\ TECHNOLOGY
}

\section{Stroboscopic effect}

\section{Citation for published version (APA):}

Perz, M., Sekulovski, D., Vogels, I., \& Heynderickx, I. (2018). Stroboscopic effect: Contrast threshold function and dependence on illumination level. Journal of the Optical Society of America A, Optics, Image Science and Vision, 35(2), 309-319. https://doi.org/10.1364/JOSAA.35.000309

DOI:

10.1364/JOSAA.35.000309

Document status and date:

Published: 01/02/2018

\section{Document Version:}

Publisher's PDF, also known as Version of Record (includes final page, issue and volume numbers)

\section{Please check the document version of this publication:}

- A submitted manuscript is the version of the article upon submission and before peer-review. There can be important differences between the submitted version and the official published version of record. People interested in the research are advised to contact the author for the final version of the publication, or visit the $\mathrm{DOI}$ to the publisher's website.

- The final author version and the galley proof are versions of the publication after peer review.

- The final published version features the final layout of the paper including the volume, issue and page numbers.

Link to publication

\section{General rights}

Copyright and moral rights for the publications made accessible in the public portal are retained by the authors and/or other copyright owners and it is a condition of accessing publications that users recognise and abide by the legal requirements associated with these rights.

- Users may download and print one copy of any publication from the public portal for the purpose of private study or research.

- You may not further distribute the material or use it for any profit-making activity or commercial gain

- You may freely distribute the URL identifying the publication in the public portal.

If the publication is distributed under the terms of Article $25 f a$ of the Dutch Copyright Act, indicated by the "Taverne" license above, please follow below link for the End User Agreement:

www.tue.nl/taverne

Take down policy

If you believe that this document breaches copyright please contact us at:

openaccess@tue.nl

providing details and we will investigate your claim. 


\title{
Stroboscopic effect: contrast threshold function and dependence on illumination level
}

\author{
Makgorzata Perz, ${ }^{1, *}$ Dragan Sekulovski, ${ }^{1}$ Ingrid Vogels, ${ }^{2}$ and Ingrid Heynderickx ${ }^{2}$ \\ ${ }^{1}$ Lighting Experience, Philips Lighting Research, High Tech Campus 7, Eindhoven, The Netherlands \\ ${ }^{2}$ Industrial Engineering \& Innovation Sciences, Eindhoven University of Technology (TU/e), Eindhoven, The Netherlands \\ ${ }^{*}$ Corresponding author: gosia.perz@lighting.com
}

Received 6 October 2017; revised 7 December 2017; accepted 28 December 2017; posted 3 January 2018 (Doc. ID 308423); published 26 January 2018

\begin{abstract}
The stroboscopic visibility measure (SVM) is a method used to quantify the stroboscopic effect visibility in general illumination application. SVM has been defined previously based on a limited number of frequencies and participants. To validate and extend SVM, five perception experiments are presented, measuring the visibility threshold of light waveforms modulated at several frequencies, conducted in two different labs. A power function is fitted through the aggregated results to develop a stroboscopic effect contrast threshold function for a "standard observer," which can be used to normalize SVM. An additional experiment shows the dependency on illumination level, extending the validity of SVM to other applications. (๑) 2018 Optical Society of America
\end{abstract}

OCIS codes: (330.4060) Vision modeling; (330.5510) Psychophysics; (330.6790) Temporal discrimination; (330.1800) Vision contrast sensitivity; (070.4790) Spectrum analysis.

\section{INTRODUCTION}

Light-emitting diodes (LEDs), a type of solid-state lighting (SSL), are a fast-evolving technology. The advantages, as well as the challenges they can pose, when used for general lighting purposes are well-documented $[1,2]$. One LED feature that can pose a challenge is the fast response to changes in the driving current, meaning that a change in the driving current is almost instantaneously translated into a change in the light output. This dynamic capability of LEDs is a clear advantage above other traditional lighting technologies for creating dynamic lighting. However, it can also lead to undesired temporal effects, such as flicker, because the current of most LED systems varies over time [3]. The most common source of current change is the supply of main power to the light source, being sinusoidally modulated at a frequency of $50 \mathrm{~Hz}$ or $60 \mathrm{~Hz}$, or modulated at $100 \mathrm{~Hz}$ or $120 \mathrm{~Hz}$, if the mains power is rectified. In addition, low frequency modulation can be introduced by disturbances in the mains power induced by additional loads on the network. Most commercially available LEDs are not directly operated using the mains power, but via a piece of electronics, called a driver, that converts the mains power into a more constant current. However, the current through the LEDs remains modulated to some extent even after the conversion, depending largely on the driver topology. Figure 1 shows three examples of the variation in light output over time when different driver topologies are applied. The light modulation can become even more pronounced, and additional unwanted lower-frequency modulations can be exhibited when dimmers are connected to LEDs [4].

Methods to suppress modulation in the light output of LEDs and, at the same time, lower the visibility of unwanted temporal effects are known. These methods, however, require compromise on the cost and efficiency, and the physical space. They also affect the lifetime of an LED system [5,6]. According to the International Commission on Illumination (CIE) light modulations can cause three different temporal light artifacts (TLAs): flicker, a stroboscopic effect, and a phantom array effect. The stroboscopic effect, which is the focus of the current study, is defined as a "change in motion perception induced by a light stimulus, the luminance or spectral distribution of which fluctuates with time, for a static observer in a non-static environment" [7]. A static observer is defined as one who does not make large saccades and, consequently, is limited to an observer making involuntary microsaccades that typically occur in normal vision. Both the stroboscopic effect and the phantom array effect are spatio-temporal effects that occur due to the movement of an image across the retina. However, it is argued that they are distinctly different. CIE defines the phantom array effect as a "change in perceived shape or spatial positions of objects, induced by a light stimulus, the luminance or spectral distribution of which fluctuates with time, for a non-static observer in a static environment." As such, the phantom array 


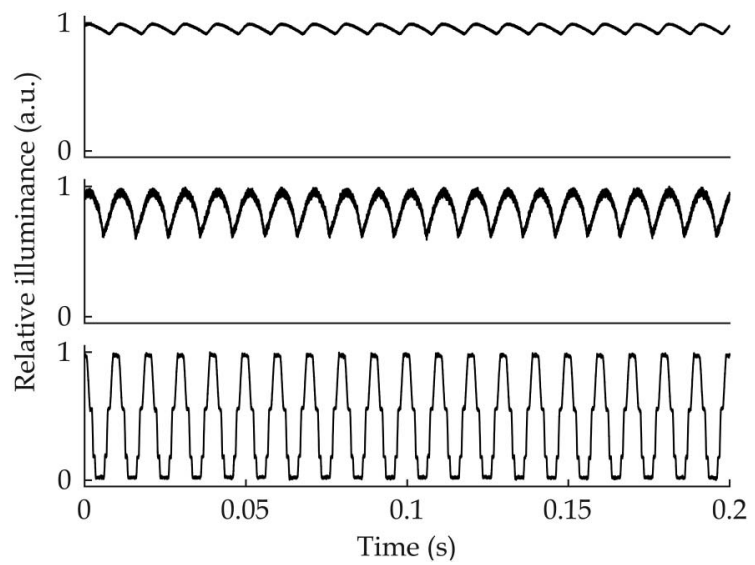

Fig. 1. Illuminance (lux) as a function of time (s) for three commercially available LED sources, using different driver topologies.

effect is caused by saccades [8], whereas the stroboscopic effect is not. Since the projected retinal images during saccades may be distorted [9], the interpretation of the retinal image may be different for the stroboscopic effect than for the phantom array effect. Evidence supporting this conclusion is given by Bedell et al. [10], who demonstrated that human observers perceive a smaller extent of motion blur when the retinal image is a result of an eye or head movement, as compared to when a moving target generates a comparable image with a stationary head and eyes. The visibility of the stroboscopic effect can be explained in terms of temporal summation, which is the capability of the human visual system to integrate information over time. Related to temporal summation is visual persistence [11]. It means that a brief stimulation of the retina results in neural activity that continues for some time after the stimulus has ceased [12]. When an object moves, the corresponding retinal image moves across the retina and activates multiple spatially distributed detectors. Because of visual persistence, this can lead to motion blur or smear for relatively fast movements. When a moving object is illuminated by modulated light, the retinal image is modulated as well. When the visual persistence time is shorter than the inverse of the frequency of the light modulation, the modulation of the retinal image can be perceived, which leads to a stroboscopic image instead of a blurred image. Hogben and Lollo [13] explained the dependence of visual persistence on luminance, showing that it increases with decreasing background levels. Consequently, at low illumination levels, the stroboscopic image merges faster into a blurred image than at higher levels. The visibility of the stroboscopic effect is thus a nonlinear function of temporal frequency and illumination level.

\section{LITERATURE OVERVIEW}

The visibility of the stroboscopic effect was first studied in relation to high-density discharge (HID) and fluorescent lamps by Eastman and Campbell [14], Frier and Henderson [15], and Rea and Ouellette [16]. A detailed overview of these studies is reported in one of our previous articles [17]. More recent studies have investigated the visibility of the stroboscopic effect in relation to light waveforms generated by LEDs. Results of a perception study, executed by Vogels et al. [18], showed that the visibility of the stroboscopic effect, in an office application, decreased with increasing modulation frequency. The visibility threshold also depended on the duty cycle of a light waveform fluctuating at $100 \mathrm{~Hz}$, following a U-shaped function. Consistent with the results of earlier studies, the visibility of the stroboscopic effect was found to be dependent on the speed of the moving object, with higher speeds producing lower visibility thresholds than lower speeds. Perception of the stroboscopic effect was subsequently studied by Bullough et al. $[19,20]$. Based on their measurements, Bullough et al. provided the relationship between frequency and modulation depth for detection and acceptability of the stroboscopic effect, though this relationship only provides meaningful data for square waveforms at $50 \%$ duty cycle and frequencies above $100 \mathrm{~Hz}$. In a subsequent paper, Bullough and Marcus [21] showed that the flicker index is a better predictor for detection and acceptability of the stroboscopic effect than the modulation depth, and they provided a modification of the flicker index, which accounts for the effect of frequency. Application of the modified flicker index is suitable when a light waveform has one frequency component with an amplitude that is significantly larger than the amplitude of all other harmonics. Later, Tu et al. [22] investigated possible cultural differences in the visibility of the stroboscopic effect for sinusoidal light waveforms at several frequencies. Their experiments showed no difference in sensitivity between Asian and European observers. Perz et al. [17] performed three perception experiments, and they developed a measure that can be used to predict the visibility of the stroboscopic effect. As an extension to the recommendations provided by Bullough et al. [20], this measure can predict the visibility of the stroboscopic effect for light waveforms of different shapes and frequencies for a given range of movement speeds in the environment. The measure, developed by Philips Lighting, was termed the Stroboscopic Visibility Measure (SVM) and it consists of a Minkowski summation of the energy in the Fourier frequency components of the light waveform, normalized for human sensitivity. It is defined as

$$
\mathrm{SVM}=\sqrt[3.7]{\sum_{m=1}^{\infty}\left(\frac{C_{m}}{T_{m}}\right)^{3.7}}\left\{\begin{array}{cc}
<1 & \text { not visible } \\
=1 & \text { just visible } \\
>1 & \text { visible }
\end{array}\right.
$$

where $C_{m}$ is the amplitude of the $m$-th Fourier component of the light waveform, and $T_{m}$ is the visibility threshold, expressed in terms of modulation depth, for a sine waveform at the corresponding frequency. Perz et al. showed that SVM is robust to normalization with either the individual visibility threshold or the threshold averaged across a group of participants. This means that, even though there might be variability across people in the visibility threshold of the stroboscopic effect, an average contrast threshold (i.e., $T_{m}$ as a function of frequency) can be used. The contrast threshold function used to establish Eq. (1) was obtained by interpolating the visibility thresholds measured for 20 observers at four frequencies, being $100 \mathrm{~Hz}$, $200 \mathrm{~Hz}, 400 \mathrm{~Hz}$, and $800 \mathrm{~Hz}$. The CIE recommends using SVM as a method to quantify the visibility of the stroboscopic effect resulting from LED sources in general illumination applications where human movements determine the highest 
velocity movements [7]. The CIE, however, also points out that further verification of SVM is needed. The contrast threshold used to define SVM was measured for one illumination level of 500 lux, measured at the task surface. This light level seems appropriate, as it closely corresponds to the light level recommended for a typical office setting (which is around 400 lux at the task area [23]). However, other applications need different illumination levels; for instance, the light level is typically much lower in outdoor, street, or road lighting. Wang et al. [24] reported results of one experiment in which the stroboscopic effect visibility thresholds of a sinusoidal light waveform modulated at $100 \mathrm{~Hz}$ were measured at several illumination levels, ranging from 5 to 500 lux. Their results showed that the visibility threshold followed a U-shaped function of the light level. The visibility thresholds were lower at illuminance levels of 30 lux, 50 lux, and 100 lux than at 5 lux, 300 lux, and 500 lux. The differences were small though, and a post-hoc test showed that, statistically, most of the conditions were not significantly different from one another. Wang et al. pointed out that the results could not be explained by using temporal and spatial contrast sensitivity functions $[25,26]$, and they emphasized that further studies are needed.

\section{GOAL OF THE CURRENT STUDY}

The aim of the current study is twofold. First, we want to improve the precision of the stroboscopic effect contrast threshold function, used for normalization in SVM, as recommended by the CIE. The new function is based on the results of five perception experiments, performed in two different labs. The visibility thresholds for sinusoidal light waveforms were measured at 10 different frequencies, for more than 70 observers in total, which provides confidence in the generalization of the results to the general population. The second goal of the study is to expand the usability of SVM into broader contexts by measuring the visibility of the stroboscopic effect at different illumination levels. We present the results of an experiment in which the thresholds of sinusoidally modulated light were measured at several different frequencies and illumination levels. These results can be used for applications, ranging from outdoor and street lighting, where the illumination levels can be as low as 10 lux, to offices and retail, where the light levels can be higher than 500 lux. The reported results provide a validation of SVM, further contributing to its standardization.

\section{EFFECT OF FREQUENCY}

To develop a more precise stroboscopic effect contrast threshold function for conditions characteristic for a typical office we combined the results of five perception experiments. Three of these experiments were previously published in $[17,22]$. Experiments 1, 2, 3, and 5 were performed in the Netherlands, and experiment 4 was performed in China. In experiments 1-4 we measured the visibility threshold, expressed in terms of modulation depth, for light waveforms sinusoidally modulated at several frequencies. As the modulation depth of a sinusoid is directly related to the amplitude of its Fourier fundamental, the results of experiments 1-4 can be directly used to normalize SVM for the corresponding frequency [i.e., $T_{m}$ in Eq. (1)]. However, sinusoids with full modulation are limited to an amplitude of their Fourier fundamental of 1 , whereas some waveforms may result in larger Fourier amplitudes. For example, for a periodic pulse train with a pulse width going to 0 , the amplitudes of all Fourier components approach a value of 2 . This implies that light waveforms with Fourier amplitudes larger than 1 still may produce a visible stroboscopic effect at frequencies for which the stroboscopic effect of a sinusoid with a full modulation cannot be detected. Thus, to extend the normalization function of SVM to higher frequencies, experiment 5 aimed to determine the highest frequency that still produces a visible stroboscopic effect for periodic pulse train light modulations. As the method used in experiments $1-4$ is different from that of experiment 5 , this section is divided into two parts.

\section{A. Experimental Method}

Because the experiments were conducted at two different locations, two identical experimental setups were custom built for each site. Understandably, the experimental procedure was the same at both sites.

\section{Setup}

A photograph of the setup is shown in Fig. 2(a). Two typical office luminaires were mounted in a frame, $0.8 \mathrm{~m}$ from each other at a height of $2.5 \mathrm{~m}$ and placed next to a white wall. Each luminaire contained five rows of cool white LEDs, having a correlated color temperature (CCT) of $6500 \mathrm{~K}$. The voltage of the LEDs was controlled by a programmable waveform generator (Agilent, 2255) via a laptop. The setup was calibrated by measuring and transforming the relation between voltage and illumination. A disk with a diameter of $27 \mathrm{~cm}$ was placed below the luminaires at a height of $75 \mathrm{~cm}$, shown in Figs. 2(b) and 2 (c). It had a black surface, having a reflectance of $7 \%$, and a white spot with a reflectance of $81 \%$ and a diameter of $2.6 \mathrm{~cm}$, placed $10 \mathrm{~cm}$ from the center of the disk. As a result, a high contrast of 12:1 was created between the target

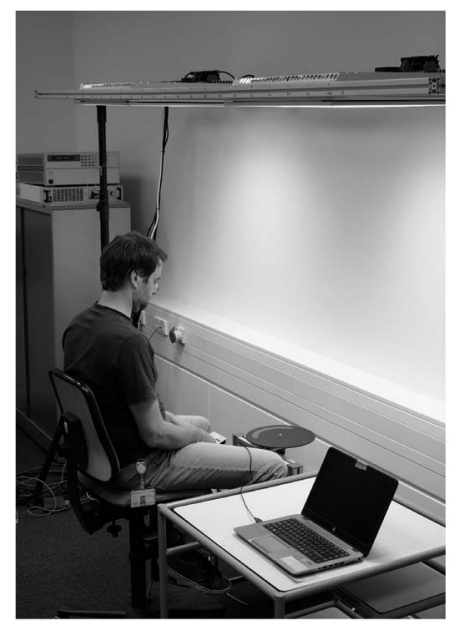

(a)

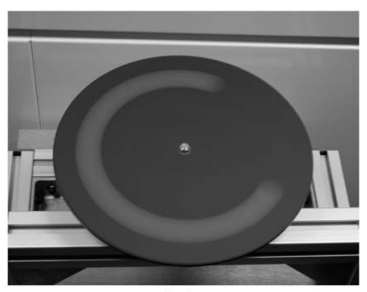

(b)

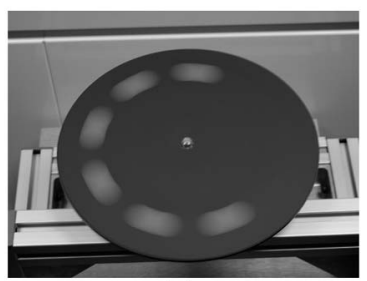

(c)
Fig. 2. (a) Picture of the setup used in the experiments, (b) an impression of the appearance of the rotating disk under constant light with no modulation, giving rise to the perception of a blurred image, and (c) the rotating disk under modulated light, resulting in a visible stroboscopic effect. 
(white spot) and the background (black surface). The timeaveraged horizontal illuminance on the disk was 500 lux. An industrial precision electromotor was used to rotate the disk at a constant speed of $4 \mathrm{~m} / \mathrm{s}$, which was chosen because it corresponds to hand movements occurring during (rapid) gestures [27]. We consider this speed as a realistic upper limit for office lighting, since the speed of other natural human movements, like walking or reaching a target, is typically lower [28-30]; and nonhuman movements with higher speeds are sporadic in offices. The dependence of the visibility of the stroboscopic effect on speed has been reported by Vogels et al. [18]. This particular experimental setup was chosen because it represents a realistic worst-case condition in a typical office environment; that is, the condition where the stroboscopic effect would be the most problematic.

\section{Procedure}

Participants were seated at $0.7 \mathrm{~m}$ from the center of the disk, which was rotating for the full course of the experiment. They read through and signed the consent form, confirming their eligibility for the study. They were told what the stroboscopic effect was and they were also presented with several examples of the effect during a short introduction to the experiment. For each stimulus during the experiment, the disk was first illuminated by a constant light output (DC) for $4 \mathrm{~s}$, after which it was illuminated by modulated light (AC). Participants were instructed to look at the rotating disk and indicate on a portable numerical keyboard whether they observed the difference between the DC and AC conditions. They were instructed to press the right arrow key if they observed the difference, meaning that they could detect the stroboscopic effect, and otherwise the left arrow key. For each light condition of an experiment, the visibility threshold was measured using an adaptive up-down method, or staircase, which is an efficient method to estimate the $50 \%$ detection threshold [31]. It would be preferred to use a two-alternative, forced-choice method (2AFC), which is typically described as "criterion-free," over the yes-no method [32], but due to hardware limitations it could not be applied. In each staircase, the starting modulation depth was set at a random value between 0.95 and 1.00 , so that the probability of detecting the stroboscopic effect was high. The modulation depth of each subsequent stimulus depended on the response of the participant to the preceding stimulus. The modulation depth was decreased if a participant observed a difference between DC and AC conditions; otherwise it was increased. The modulation depth at which the answer changed from positive ("I see the difference") to negative ("I don't see the difference"), or vice versa, was counted as a reversal point, and eight such reversal points were measured for each light waveform condition. The visibility threshold was obtained as the arithmetic mean of the last four reversal points. As such, the visibility threshold corresponded to the modulation depth at which a participant reported detection with a probability of $50 \%$. All staircase stimuli were intermingled and presented in a random order, different for each participant.

\section{Light Conditions}

In experiment 1 , the visibility thresholds of light waveforms sinusoidally modulated at $50 \mathrm{~Hz}, 100 \mathrm{~Hz}, 200 \mathrm{~Hz}$, and $400 \mathrm{~Hz}$ were measured twice per participant. The arithmetic mean of the two measured values was used as the resulting threshold. In experiment 2, the visibility thresholds were measured for both sinusoidally modulated light waveforms and square waveforms at $50 \mathrm{~Hz}, 100 \mathrm{~Hz}, 200 \mathrm{~Hz}$, and $400 \mathrm{~Hz}$. In experiment 3 , earlier data of the sinusoidal light waveforms at $100 \mathrm{~Hz}, 200 \mathrm{~Hz}$, and $400 \mathrm{~Hz}$ were repeated and extended with $800 \mathrm{~Hz}$. Finally, in experiment 4, light waveforms sinusoidally modulated at frequencies of $100 \mathrm{~Hz}, 150 \mathrm{~Hz}, 200 \mathrm{~Hz}$, $250 \mathrm{~Hz}, 300 \mathrm{~Hz}, 400 \mathrm{~Hz}, 500 \mathrm{~Hz}, 750 \mathrm{~Hz}$, and $800 \mathrm{~Hz}$ were used to thoroughly sample the relation between the visibility of the stroboscopic effect and frequency of the sinusoidally modulated light. In each of the experiments, a full-factorial within-subject design was used. The first three experiments took about 30 min per participant, and the fourth experiment took about $45 \mathrm{~min}$.

\section{Participants}

In accordance with the regulations of the internal ethical committee of Philips Lighting, participants who might have oversensitivity to temporal modulated light were excluded from the experiments. Hence, participants who were included in the study did not suffer from epilepsy nor had a family history of epilepsy, and did not suffer from migraines. In experiments 1 , 2, and 3, the observers were mostly Western European; in experiment 4, they were Asian. In the first experiment, 12 participants measured their visibility threshold: seven males and five females, with ages ranging from 18 to 38 years. In the second experiment, 20 people participated: 13 males and seven females, with ages ranging from 19 to 33 years. The third experiment was completed by 20 participants: 12 males and eight females, with ages ranging from 19 to 36 years. In the fourth experiment, 21 participants took part: 10 females and 11 males, with ages ranging from 21 to 47 . Thus, a proper balance in the participants' gender was maintained over all experiments. Their relatively young age was appropriate since our goal was to define a measure for a "standard observer" with healthy vision.

\section{B. Results}

The visibility thresholds were measured in terms of modulation depth in all four experiments. The results of the first experiment are shown in Fig. 3 as violin plots and error bars. A violin plot is a combination of a box plot and density traces (or a smoothed histogram) into a single diagram [33]. In Fig. 3, the horizontal solid and dotted lines in each plot correspond to the mean and median threshold values, respectively. The lower and upper borders of the darker shaded areas correspond to the 25th and 75 th percentiles. The error bars (i.e., the vertical lines) show the $95 \%$ confidence interval (CI) of the mean.

Figure 3 shows that the visibility threshold increases with the increasing frequency of the light modulation. At $50 \mathrm{~Hz}$, the visibility threshold is lowest with a mean modulation depth of 0.20 ; at $400 \mathrm{~Hz}$, the threshold is highest with a mean modulation depth of 0.52 . The violin plot at $400 \mathrm{~Hz}$ clearly exhibits a considerable difference between the mean and median threshold values, being 0.52 and 0.42 , respectively. This is because the threshold values are not normally distributed, as indicated by the shape of the violin plot: part of the data clusters around a threshold value of 0.38 , whereas a smaller part of the data clusters around a threshold value of 0.88. An ANOVA, performed 


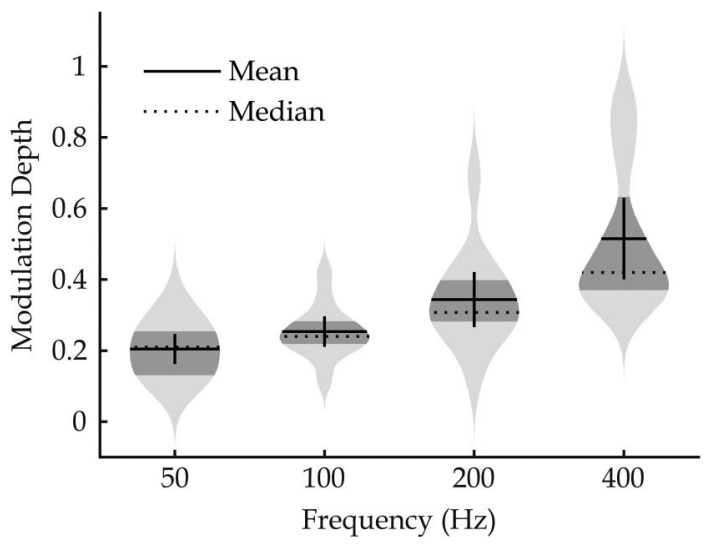

Fig. 3. Violin plots of the visibility thresholds, expressed as modulation depth, as a function of modulation frequency, measured in experiment 1; the mean and median values are depicted as solid and dotted lines, respectively; the borders of the dark shaded areas mark the 25th and 75th percentiles; and the error bars correspond to the $95 \%$ CI of the mean.

with modulation depth as a dependent variable, frequency as an independent variable, and participant as a random factor, demonstrated that, as expected, the modulation frequency had a significant effect on the visibility of the stroboscopic effect, $F(3,47)=30.5, p<0.001, \omega=0.42$. The effect of the participant was also significant $(p<0.001)$. The results of the subsequent three experiments are visualized and analyzed in the same way, and shown in Fig. 4. The top-left and top-middle graphs in Fig. 4 show the visibility thresholds measured in experiment 2 for sinusoidally and squared modulated light waveforms, respectively. The thresholds of the square light waveforms are corrected to make them comparable to those of the sinusoidal waveforms. Perz et al. [17] demonstrated that the ratio of the stroboscopic effect visibility threshold of a square over a sinusoidal light waveform at the same fundamental frequency equals the inverse of the amplitude of the fundamental frequency component of the square waveform. Therefore, by dividing the visibility thresholds of the square waveforms by the $\pi / 4$ (i.e., the fundamental component of a square waveform), the thresholds of sinusoidal waveforms at the corresponding frequency are obtained. A paired sample $t$-test was conducted to compare the visibility thresholds of these two types of light waveforms (over all modulation frequencies), and it showed that there is no significant difference in the visibility threshold between the sinusoidal and the corrected square waveforms $[t(79)=0.26, p=0.8$, and Hedges' $g=0.012$ ]. The violin plots of experiment 2 show that the lowest mean visibility threshold was measured at $50 \mathrm{~Hz}$ and it equals to 0.28 . The highest mean threshold corresponds to a modulation depth of 0.60 measured at $400 \mathrm{~Hz}$. The two graphs also show that data is not normally distributed; the distribution in visibility thresholds seems bimodal, especially at the highest frequency of $400 \mathrm{~Hz}$. The top-right and bottom graphs show the visibility thresholds measured in experiment 3 and 4, respectively. At the lowest measured frequency of $100 \mathrm{~Hz}$, the visibility threshold corresponds to 0.26 in experiment 3 , and to 0.23 in experiment 4 . As in the previous two experiments, these thresholds increase with increasing frequency. At a modulation frequency of $800 \mathrm{~Hz}$ in experiment 3 and at $750 \mathrm{~Hz}$ and $1000 \mathrm{~Hz}$ in experiment 4 , the violin plots are remarkably narrow, and the means and medians are approximately 1.00. Analysis of staircases per participant showed that nearly all were clipped at the upper boundary. This means that the stroboscopic effect produced by a full modulation sinusoidal waveform is not visible for most (a)

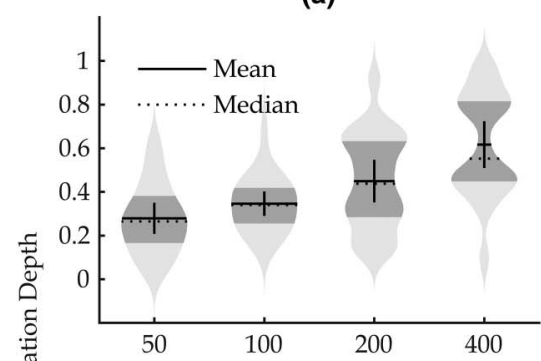

$\frac{\frac{\pi}{3}}{\frac{\pi}{3}}$

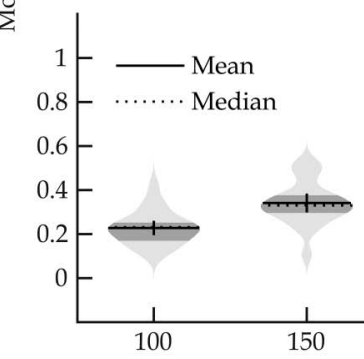

(b)

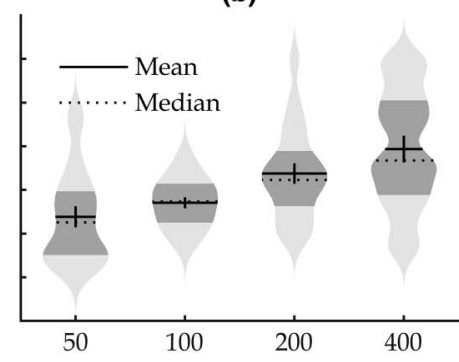

(d)

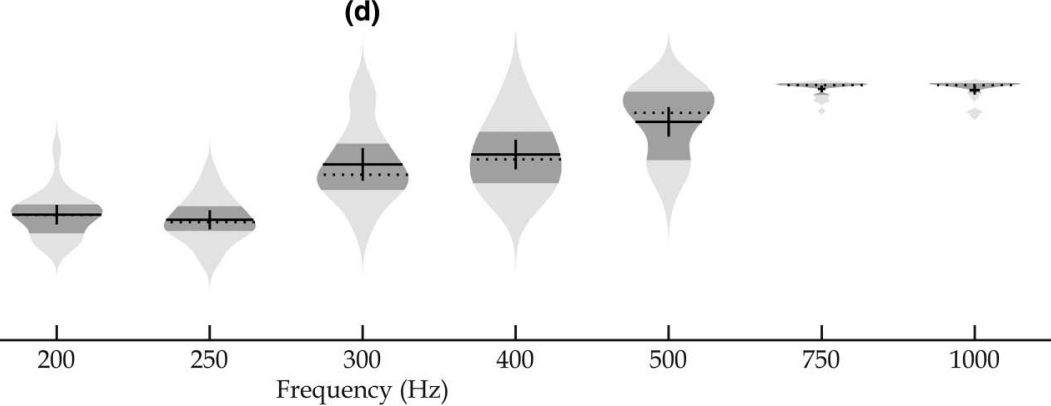

(c)

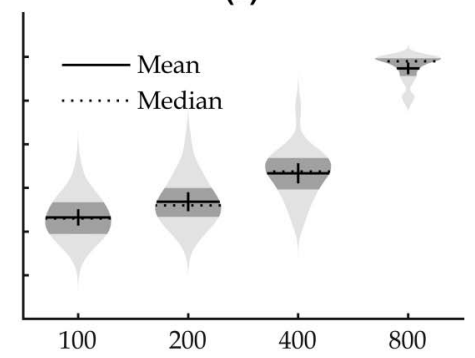


observers in light modulated at frequencies above $750 \mathrm{~Hz}$. For each experiment an ANOVA showed that the visibility of the stroboscopic effect depends on both the frequency of the modulated light $(p<0.005)$ and the participant $(p<0.001)$.

Results of experiments $1-4$ can be used for normalization in SVM for frequencies up to about $750 \mathrm{~Hz}$. Above this frequency, illuminating the rotating disk by sinusoidal light waveforms did not produce a visible stroboscopic effect. To determine the normalization function above $750 \mathrm{~Hz}$, stroboscopic effect detection using periodic pulse train light waveforms was measured, and the waveform at the highest frequency, producing the visibility threshold effect was determined.

\section{Experimental Method}

The same setup, as in experiments 1-4, was used (see Fig. 2). The participants' task was also the same as in the previous experiments, but a constant stimuli method rather than the staircase method was used. This implies that each participant saw all light stimuli, repeated 3 times, in random order. The visibility threshold was then determined as the frequency at which the stroboscopic effect was detected $50 \%$ of the time across all observations (per frequency). Nine observers took part in the experiment; three were female and six were male, and their ages ranged from 27 to 52 years. Section 4.A.4 describes the inclusion criteria. The light stimuli included the periodic pulse train waveforms with a full modulation depth at a 5\% duty cycle. Using waveforms with a smaller duty cycle, and so with a larger amplitude of the Fourier components, was not possible due to hardware limitations. These pulse trains were modulated at eight frequencies ranging from $800 \mathrm{~Hz}$ to $1500 \mathrm{~Hz}$, in steps of $100 \mathrm{~Hz}$. The average illuminance, measured on the rotating disk, was just above 200 lux. This was dimmer compared to the 500 lux level that was used in experiments 1-4. However, as we will show in Section 6, thresholds measured for illuminances of 100 lux and 500 lux are not different from each other.

\section{Results}

The probability of detecting the stroboscopic effect was aggregated across participants at each frequency, and the results are shown in Fig. 5. At frequencies of $800 \mathrm{~Hz}$ and $900 \mathrm{~Hz}$, the stroboscopic effect was almost always visible; at frequencies of $1200 \mathrm{~Hz}$ and above, however, the effect was almost never detected. A generalized linear regression model with a probit link function was fitted through the detection probabilities as a function of frequency, and the computed predicted probability values are plotted in Fig. 5 as solid line. The resulting visibility threshold, which corresponds to a $50 \%$ probability of detecting the stroboscopic effect, was found at a frequency of $1110 \mathrm{~Hz}$.

\section{STROBOSCOPIC EFFECT CONTRAST THRESHOLD FUNCTION}

The results of all five experiments are aggregated to determine the stroboscopic effect contrast threshold function, expressed in terms of the amplitude of the Fourier frequency component, denoted as $T_{m}$ in Eq. (1). The visibility thresholds, measured

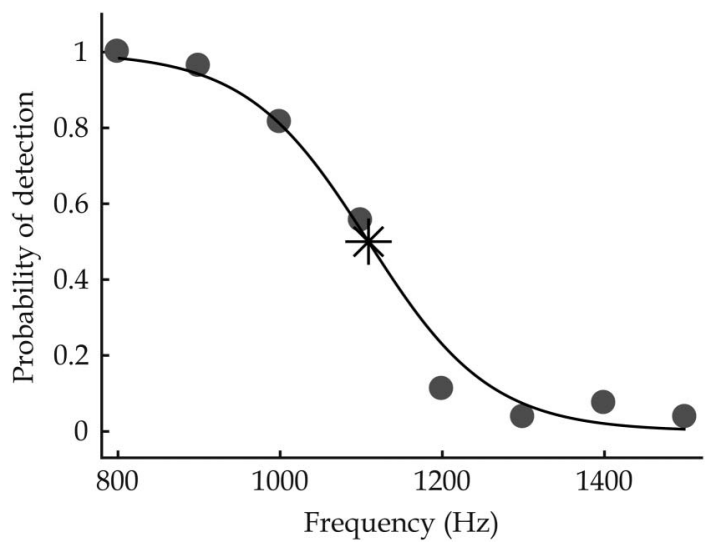

Fig. 5. Mean probability of the stroboscopic effect detection as a function of frequency of periodic pulse waveforms. The solid line depicts predicted detection values computed for the generalized linear model with probit link function. The star depicts the visibility threshold at a frequency of $1110 \mathrm{~Hz}$.

for sinusoidal light waveforms in experiments $1-4$, up to and including $500 \mathrm{~Hz}$, are used. The results for frequencies above $750 \mathrm{~Hz}$ are discarded, since at these frequencies the stroboscopic effect could not be detected. As the violin plots indicate that some of the data is not normally distributed (see Fig. 4), median rather than mean visibility thresholds are further used. These results are presented in Fig. 6. In addition, the visibility threshold of a pulse train modulation with a frequency of $1110 \mathrm{~Hz}$ from experiment 5 is included. Having no a priori knowledge to assume any specific shape of the contrast threshold function, three different functions are evaluated, being an exponential function $\left[T(f)=a * e^{(b * f)}\right]$, a power function $\left[T(f)=a * f^{b}+c\right]$, and a logarithmic function $\left[T(f)=a+b * \log _{2}(f+c)\right]$. At the visibility threshold SVM, defined in Eq. (1), should equal to 1 . Thus, we used the absolute difference between 1 and the SVM values, computed for the waveforms at threshold, as the minimization criterion, and optimized the parameters of the three functions accordingly, using the fminsearch function in Matlab, based on the Nelder-Mead simplex algorithm [34]. With the optimized functions, we computed SVM for the waveforms at threshold, and used the root mean square error (RMSE), defined as the square root of the mean squared residuals between 1 and the computed SVM values to determine the overall best function. The RMSE equals 0.17 for the exponential function, 0.15 for the power function, and 0.18 for the logarithmic function. Moreover, the power function most accurately predicts the visibility threshold of a waveform with a complex spectrum, with a fundamental at $1110 \mathrm{~Hz}$ (exponential, SVM $=0.69$; power, $S V M=1.00$; and logarithmic, $S V M=1.26$ ). This power function is plotted in Fig. 6(a) as a solid line, together with the results of experiments $1-4$, averaged across observers per frequency and per experiment. The black dot at a relative amplitude of 2 with the corresponding frequency of $1280 \mathrm{~Hz}$ depicts the upper frequency limit of the visibility of the stroboscopic effect of a light waveform with only one fundamental frequency, under conditions used in the experiment. The 


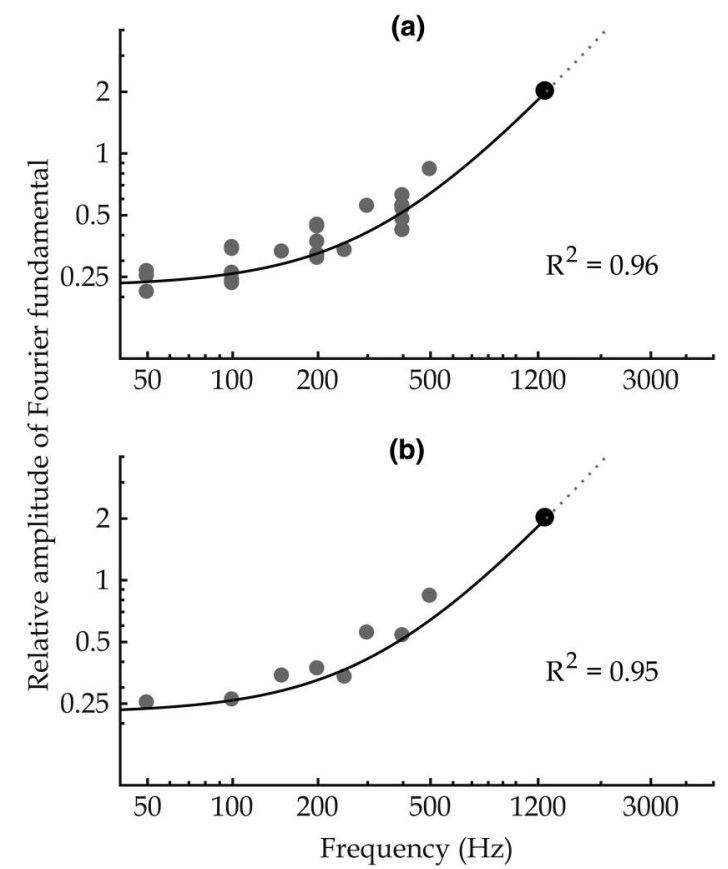

Fig. 6. Log-log plot of the median visibility threshold expressed in terms of the amplitude of Fourier fundamental, combined over experiments 1 to 4 , and averaged across participants per frequency, in (a) per experiment, and in (b) over all four experiments. The solid line corresponds to the power function, as defined in Eq. (2). The black dot depicts the highest frequency at which the stroboscopic effect can be detected.

contribution of the harmonics is negligible, given that they are at much higher frequencies.

The stroboscopic effect contrast threshold function is given by

$$
T(f)=2.865 * 10^{-5} * f^{1.543}+0.225 .
$$

To further assess the goodness-of-fit, the coefficient of determination, $R^{2}$, was calculated as one minus the ratio of the sum of squares of the residuals to the total sum of squares, between the contrast threshold function and median visibility thresholds of sinusoids. It yielded a $R^{2}$-value of 0.96 , indicating that $96 \%$ of the variability in the median visibility threshold of sinusoidally modulated light is explained by frequency. The large value of $R^{2}$ indicates that the function, as defined in Eq. (2), is a good representation of the median visibility threshold as a function of the modulation frequency of a sinusoidal light waveform. The visibility thresholds can also be averaged across observers' responses per frequency, over all experiments combined. By doing so, we obtained one threshold value per frequency, which are plotted in Fig. 6(b), together with the contrast threshold function, defined in Eq. (2). The $R^{2}$ between the median threshold values and the normalization curve were computed to be 0.95 . This shows that the shape of the curve is robust to averaging per frequency, either per experiment or over all experiments. Therefore, it can be concluded that the stroboscopic effect contrast threshold function, as defined in Eq. (2), is an adequate representation of the visibility threshold of a "standard observer" to the stroboscopic effect.

\section{EFFECT OF ILLUMINATION LEVEL}

The SVM, used to quantify the visibility of the stroboscopic effect, has been determined in conditions typical for an office, with an averaged illuminance of 500 lux at the task surface. The contrast threshold function, reported in Section 6, was measured at the same illumination level, and it can be used for normalization in SVM to predict the visibility of the stroboscopic effect in applications with a comparable illumination level. The illumination level, though, can vary considerably across applications; thus, the validity of SVM for different illumination levels needs to be determined. Results of the experiment, reported by Wang et al. [24], show that the visibility threshold for sinusoidal modulations at $100 \mathrm{~Hz}$ follows a U-shaped function of light level; thresholds were highest at 5 lux (i.e., 0.31) and 500 lux (i.e., 0.28), while the lowest threshold was about 0.21 , measured at 50 lux. Wang et al. concluded that further studies were needed to understand the effect of the illumination level on the visibility of the stroboscopic effect at different frequencies. The current experiment has been conducted to validate the results reported by Wang $e$ t al. by measuring the visibility thresholds of light waveforms sinusoidally modulated at $100 \mathrm{~Hz}$ at a subset of the illumination levels used in their study. Further, the experiment extended the results of Wang et al. by measuring visibility thresholds of waveforms modulated at four frequencies at one illumination level of 50 lux.

\section{A. Experimental Method}

The experiment was executed using the same setup and following the same procedure as described in Section 4.

\section{Light Conditions}

Sinusoidally modulated light waveforms with modulation depths ranging between 0 and 1.00 were used as stimuli. Nine light conditions were presented with different values for the modulation frequency and illumination level. For a frequency of $100 \mathrm{~Hz}$, five levels of horizontal illuminance at the rotating disk were presented, namely 5 lux, 10 lux, 50 lux, 100 lux, and 500 lux. For an illumination level of 50 lux, four frequencies were presented: $100 \mathrm{~Hz}, 200 \mathrm{~Hz}, 400 \mathrm{~Hz}$, and $800 \mathrm{~Hz}$. The nine conditions were divided over five blocks such that the illumination level was constant during one block. The corresponding stimuli were all intermingled and randomized. The presentation order of the blocks was randomized per participant. To ensure that participants were naïve to the staircase procedure and lower the fatigue due to repeating near the threshold stimuli, additional stimuli at the same illumination level with a large modulation depth (i.e., between 0.80 and 1.00 ) were randomly intermingled into each block. Two minutes of adaptation time was included between the blocks; it was previously demonstrated that it is a sufficient adaptation time for the chosen illumination levels [35]. The experiment took about $40 \mathrm{~min}$ per person.

\section{Participants}

Visibility thresholds of 17 participants were measured: five females and 12 males, with ages ranging from 19 to 46 years. Similar to experiments 1 to 4 , we followed the regulations of 
the internal ethical committee of Philips Lighting when choosing participants.

\section{B. Results}

The measured visibility thresholds, expressed as modulation depths, are shown as violin plots in Fig. 7(a) for sinusoidal waveforms modulated at $100 \mathrm{~Hz}$ at several illumination levels, and in Fig. 7(b) for sinusoidal waveforms with a horizontal illuminance of 50 lux at the task surface, modulated at several frequencies. Figure $7(\mathrm{a})$ shows that the mean threshold was highest at the lowest illumination level of 5 lux, where it was 0.40 . The shape of the corresponding violin plot indicates that there was a large variation between participants with a skew to the upper threshold values. The mean visibility threshold then decreased up to an illumination level of 50 lux, where it reached the lowest value of 0.18 . For higher illumination levels, the mean visibility threshold increased again, corresponding to a modulation depth of 0.23 at 100 lux and 0.27 at 500 lux. An ANOVA, performed with modulation depth as a dependent variable, the illumination level as independent variable, and participant as random factor, confirmed that the visibility of the stroboscopic effect depended on the illumination level, $F(4,84)=17.9 p<0.001, \omega=0.35$; and the effect of the
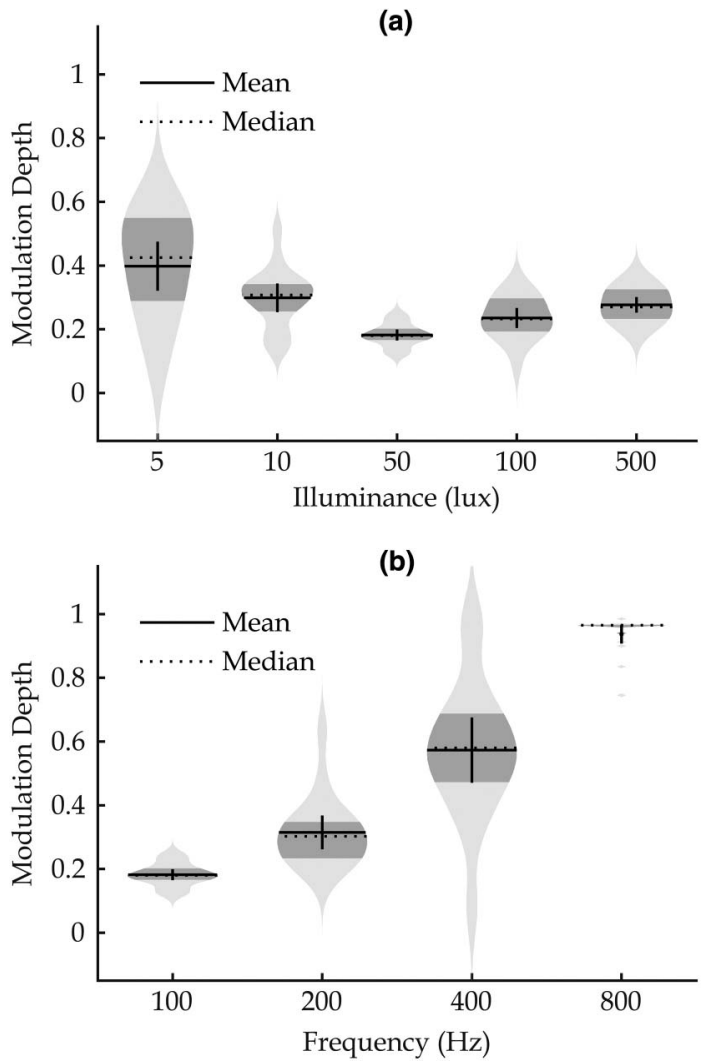

Fig. 7. Violin plots of visibility thresholds, expressed in modulation depth, for (a) a sinusoidal waveform at $100 \mathrm{~Hz}$ measured at different illumination levels, and (b) a sinusoidal waveform with a horizontal illumination of 50 lux at the task surface measured at different frequencies. The mean and median threshold values are depicted as solid and dotted lines, respectively. The borders of the darker shaded areas mark the 25th and 75 th percentiles. The error bars correspond to the $95 \% \mathrm{CI}$ of the mean. participant was significant $(p<0.001)$. Post-hoc comparisons using $t$-tests with Bonferroni correction, showed that the highest threshold at 5 lux was significantly different from the thresholds at all other illumination levels, and the lowest threshold at 50 lux was significantly different from all other thresholds, except for the one at 100 lux.

Figure 7(b) shows that, for a sinusoidal waveform with an illumination of 50 lux at the task surface, the visibility threshold increased with increasing frequency, as expected. The lowest threshold of 0.18 was measured at a modulation frequency of $100 \mathrm{~Hz}$. At a frequency of $800 \mathrm{~Hz}$, the violin plot became very narrow, and the mean and median approached 1.00 , meaning that, at this frequency, the stroboscopic effect couldn't be detected anymore by most of the observers. An ANOVA confirmed a significant effect of frequency on the visibility of the stroboscopic effect at an illumination level of 50 lux, $F(3,67)=159.3, p<0.001, \omega=0.84$, and a significant effect of participant $(p<0.01)$. Post-hoc comparisons with Bonferroni correction showed that the visibility threshold at all frequencies were significantly different from one another.

\section{COMPARISON OF RESULTS FROM DIFFERENT EXPERIMENTS}

The results of all experiments on the visibility of the stroboscopic effect (using the same setup with the rotating disk as stimulus) can be combined to test if the results are consistent. These include the experiments reported in $[17,22,24]$ and in Section 6. Consistency in results proves the accuracy of the threshold values and validates the reliability of the methodology used to measure them. The latter is of particular importance, as the yes-no procedure that was employed is known to depend on participants' response criterion [36-38], which can also be culture-dependent. First, the visibility threshold of sinusoidally modulated light at a frequency of $100 \mathrm{~Hz}$ and a horizontal illumination of 500 lux at the task surface can be compared for the five experiments reported in this paper. To test the equality in the mean of the visibility threshold over the experiments, an ANOVA was performed with visibility threshold as a dependent variable and experiment as an independent variable. The thresholds of the sinusoidal and those corrected to sinusoidal square waveforms, which were obtained in experiment 2 , were averaged. It should be noted that the number of threshold measurements varied per experiment, and unequal sample sizes can affect the required assumption of homogeneity of variance in ANOVA. ANOVA is considered robust to moderate deviations from this assumption, and since the number of threshold measurements was comparable in each experiment, performing an ANOVA was deemed appropriate. It showed no significant effect of experiment on the visibility threshold, expressed in modulation depth, $F(3,84)=0.45, p=0.72, \omega=-0.02$. Hence, we can conclude that for this frequency and illumination level, the measured thresholds are consistent across different experiments. Moreover, because the experiments were conducted partly in Europe and partly in China, we can also conclude that the visibility of the stroboscopic effect is culture-independent.

Second, the visibility threshold at an illumination of 50 lux at the task surface can be compared to 500 lux for several 


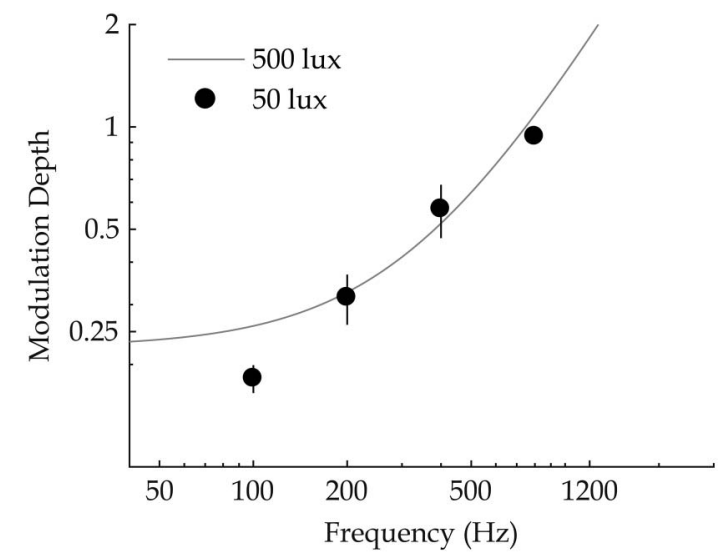

Fig. 8. Comparison of the visibility of the stroboscopic effect for different illumination levels at the task surface; the solid line shows the contrast threshold function, defined in Eq. (2), obtained for an illumination level of 500 lux, whereas the dots correspond to mean visibility threshold measured at four frequencies for an illumination level of 50 lux. The error bars correspond to the $95 \% \mathrm{CI}$ of the mean.

frequencies. Results reported in Section 6 show that the visibility threshold of sinusoidally modulated light at $100 \mathrm{~Hz}$ is significantly lower for an illumination of 50 lux than the threshold for an illumination of 500 lux. Since we measured the visibility threshold at 50 lux at four different frequencies $(100 \mathrm{~Hz}, 200 \mathrm{~Hz}, 400 \mathrm{~Hz}$, and $800 \mathrm{~Hz})$, these values can be compared to the stroboscopic effect contrast threshold function, measured at 500 lux [as defined in Eq. (2)]. The results of this comparison are shown in Fig. 8. It can be seen that the visibility threshold for a sinusoidal modulation at $100 \mathrm{~Hz}$ is different at 50 lux from the sensitivity measured at 500 lux. At higher frequencies, the sensitivity curve determined at 500 lux falls within the error bars of the mean threshold values measured at 50 lux, suggesting that they are not significantly different from each other. At $800 \mathrm{~Hz}$, the stroboscopic effect was no longer detected (see the violin plot in Fig. 7), so the results cannot be compared. T-tests were conducted, confirming a significant difference at $100 \mathrm{~Hz}[t(16)=8.80$, $p<0.001$, Hedges' $g=2.03$ ], but no significant difference at $200 \mathrm{~Hz}[t(16)=0.39, p=0.70]$, and at $400 \mathrm{~Hz}[t(16)=$ $1.10, p=0.32]$.

Finally, we compared the results reported in the current study in Section 6 to those reported earlier by Wang et al. [24]. In both studies, the visibility thresholds followed a U-shaped function of illuminance. The lowest thresholds were measured at an illumination level of 50 lux, corresponding to a modulation depth of 0.18 in experiment 5 of the current study and to 0.19 in the study of Wang et al. The small increase in visibility threshold for illumination levels above 50 lux is also similar in both studies, reaching 0.28 in experiment 5 and 0.26 in the study of Wang et al. at 500 lux. At the low illumination levels of 5 and 10 lux the results of the two studies differ, with higher thresholds of 0.40 and 0.30 measured in experiment 5 compared to 0.31 and 0.24 in the study of Wang et al. Despite these differences in absolute value, it is apparent that in both studies the visibility threshold is significantly higher at these low illumination levels than at 50 lux. In the latter respect, the results of the two studies are consistent. Possible explanations for the deviation in absolute values are discussed in the next section.

\section{GENERAL DISCUSSION}

The current paper reports the results of five perception experiments that were conducted to better understand the visibility of the stroboscopic effect, and to simultaneously increase the accuracy and validity of the SVM. In the first part of this paper, the results of five experiments were used to develop the stroboscopic effect contrast threshold function, corresponding to the sensitivity of a "standard observer." We showed that the shape of the curve is robust to averaging the visibility threshold per frequency across participants for each experiment or across all participants of all experiments. In other words, even though there is variability across the experiments and people in threshold modulation depth for the stroboscopic effect, an average threshold function can be used to represent the general population. Spread within the general population is obvious from the bimodal distribution that we found for the measured visibility thresholds in some conditions; for instance, at a frequency of $400 \mathrm{~Hz}$ measured in the second experiment (the results are shown in Fig. 4). There may be two reasons for such a spread. First, the bimodal distribution may be caused by the fact that participants used a different strategy while executing the experiments. It has been shown before that the yes-no methodology we used in our experiments might sometimes lead to biased estimates that may also be participant dependent [36-38]. But since we offered a thorough and comprehensive explanation of the experimental procedure to every participant, and interleaved stimuli of different staircases, we consider the effect of a different strategy over participants limited. Second, it is plausible that the participants in the experiments may have different spatiotemporal sensitivities. Sensitivity to the stroboscopic effect, like a sensitivity to other visual percepts, may depend on several individual characteristics, such as age or gender, but may also depend on external factors, such as time of day $[39,40]$. To determine how exactly sensitivity to the stroboscopic effect varies with individual characteristics and external factors is beyond the scope of this paper, but does need additional study. As such, the current study should not be interpreted as providing a contrast threshold function for all uses, including very sensitive participants; it proposes a function for a "standard observer," which is useful to define general guidelines for the design of waveform-modulated LEDs. The last experiment reported in this paper shows the dependency of the stroboscopic effect visibility on the illumination level. The visibility threshold of sinusoidally modulated light at $100 \mathrm{~Hz}$ at a low illumination level of 5 and 10 lux was found to be significantly higher than the visibility threshold at 500 lux. We are cautious to provide a definitive physiological explanation for this difference in the visibility threshold, but attribute it to either a decrease in visual acuity or an increase in visual persistence time at the lower illumination levels, or, presumably, the combination of these two aspects. It is known that scotopic vision (i.e., vision under low light levels) is produced through rod, rather than cone, cells, and the resulting spatial resolution is much lower as compared to photopic vision 
(i.e., vision under more standard light levels). Light levels of 5 lux and 10 lux approach mesopic vision, where both rods and cones are involved. Moreover, participants observed the moving stimulus with their fovea, where rods are absent. Hence, the visual acuity of the observers might be reduced at 5 and 10 lux, which would lead to increased visibility thresholds for the stroboscopic effect. The second possible explanation is linked to research conducted for the visibility of temporal effects in displays, where it was found that the duration of smear (i.e., visual persistence) produced by a point in apparent motion increased with decreasing background luminance levels [13]. A low luminance level of $0.3 \mathrm{~cd} / \mathrm{m}^{2}$ produced a significantly longer smear, reaching $70 \mathrm{~ms}$, compared to a luminance level of $30 \mathrm{~cd} / \mathrm{m}^{2}$, at which the maximal smear was around $45 \mathrm{~ms}$. This result would imply that at a low illumination level of 5 or 10 lux, the visual persistence time is longer. Hence, at such low illumination levels, the stroboscopic image merges into a blurred image for higher modulation depths than at higher illumination levels. The dependence on the illumination level has implications for LED design for applications such as outdoor, street, or road lighting. As people are less sensitive to light modulations at low illumination levels, the recommended limits in allowable waveform modulation can be looser as compared to limits in other applications, such as an office. It should be noted that the definition of SVM fully focuses on the visibility of the stroboscopic effect. Being visible does not necessarily mean that the effect is also problematic in real-life applications. Measuring acceptability of the stroboscopic effect could result in recommendations for light modulations in which the effect is slightly visible. Measuring acceptability, though, is not straightforward, since it is affected by several parameters, including (but probably not limited to) type of the visual tasks [41], their relative importance and corresponding speed of movements $[42,43]$, as well as the exposure duration [43]. CIE recommends using SVM to quantify visibility of the stroboscopic effect in general lighting applications with illuminations of 100 lux and above. The contrast threshold function, developed in the current study can be used for normalization in SVM. By doing so, the SVM predictions represent a "standard observer," including different cultural backgrounds. In addition, we recommend that the function, measured at 50 lux, is used to extend the validity of SVM to applications with lower illumination levels at the task surface. Additional measurements are needed, however, for illumination levels approaching scotopic vision.

Funding. Philips Lighting Research, regular project activities.

Acknowledgment. We thank Lili Wang and Yan Tu from Southeast University for their contributions.

\section{REFERENCES}

1. E. F. Schubert and J. K. Kim, "Solid-state light sources getting smart," Science 308, 1274-1278 (2005)

2. A. De Almeida, B. Santos, B. Paolo, and M. Quicheron, "Solid state lighting review-potential and challenges in Europe," Renew. Sustain. Energy Rev. 34, 30-48 (2014).
3. C. Branas, F. J. Azcondo, and J. M. Alonso, "Solid-state lighting: a system review," IEEE Ind. Electron. Mag. 7(4), 6-14 (2013).

4. D. Rand, B. Lehman, and A. Shteynberg, "Issues, models and solutions for triac modulated phase dimming of LED lamps," in Power Electronics Specialists Conference (IEEE, 2007), pp. 1398-1404.

5. M.-H. Chang, D. Das, P. Varde, and M. Pecht, "Light emitting diodes reliability review," Microelectron. Reliab. 52, 762-782 (2012).

6. M. Arias, A. Vázquez, and J. Sebastián, "An overview of the AC-DC and DC-DC converters for LED lighting applications," Automatika 53, 156-172 (2012).

7. CIE, "Visual aspects of time-modulated lighting systems-definitions and measurement models," CIE TN 006:2016 (2016).

8. W. A. Hershberger and J. S. Jordan, "The phantom array: a perisaccadic illusion of visual direction," Psychol. Rec. 48, 21-32 (1998).

9. E. Kowler, "Eye movements: the past 25 years," Vision Res. 51, 1457-1483 (2011).

10. H. E. Bedell, J. Tong, and M. Aydin, "The perception of motion smear during eye and head movements," Vision Res. 50, 2692-2701 (2010).

11. D. C. Burr, "Temporal summation of moving images by the human visual system," Proc. R. Soc. London B 211, 321-339 (1981).

12. H. L. Hawkins and G. L. Shulman, "Two definitions of persistence in visual perception," Atten. Percept. Psycho. 25, 348-350 (1979).

13. J. H. Hogben and V. Di Lollo, "Suppression of visible persistence in apparent motion," Atten. Percept. Psycho. 38, 450-460 (1985).

14. A. A. Eastman and J. H. Campbell, "Stroboscopic and flicker effects from fluorescent lamps," Illum. Eng. 47, 27-35 (1952).

15. J. P. Frier and A. Henderson, "Stroboscopic effect of high intensity discharge lamps," J. Illum. Eng. Soc. 3, 83-86 (1973).

16. M. S. Rea and M. Ouellette, "Table-tennis under high intensity discharge (HID) lighting," J. Illum. Eng. Soc. 17, 29-35 (1988).

17. M. Perz, I. M. L. C. Vogels, D. Sekulovski, L. Wang, Y. Tu, and I. E. J. Heynderickx, "Modeling the visibility of the stroboscopic effect occurring in temporally modulated light systems," Light. Res. Technol. 47, 281-300 (2015)

18. I. M. L. C. Vogels, D. Sekulovski, and M. Perz, "Visible artefacts of LEDs," in 27th Session of the CIE, Sun City, South Africa (CIE, 2011)

19. J. D. Bullough, K. S. Hickcox, T. R. Klein, and N. Narendran, "Effects of flicker characteristics from solid-state lighting on detection, acceptability and comfort," Light. Res. Technol. 43, 337-348 (2011).

20. J. D. Bullough, K. S. Hickcox, T. R. Klein, A. Lok, and N. Narendran, "Detection and acceptability of stroboscopic effects from flicker," Light Res. Technol. 44, 477-483 (2012).

21. J. D. Bullough and D. Marcus, "Influence of flicker characteristics on stroboscopic effects," Light. Res. Technol. 48, 857-870 (2016).

22. Y. Tu, L. Wang, J. Zhang, F. Lu, L. Liu, M. Perz, and I. M. L. C. Vogels, "Cross-cultural similarities in the visibility of the stroboscopic effect," in 10th China International Forum on Solid State Lighting (ChinaSSL) (IEEE, 2013), pp. 170-173.

23. "Light and lighting-lighting of work places-part 1: indoor work places," Standard: CEN-EN 12464-1 (2011).

24. L. Wang, Y. Tu, J. Zhang, F. Lu, L. Liu, I. M. L. C. Vogels, M. Perz, and I. E. J. Heynderickx, "Influence of illumination level on the visibility of the stroboscopic effect," in 7th Lighting Conference of China, Japan and Korea (CJK, 2014).

25. D. H. Kelly, "Visual responses to time-dependent stimulil. amplitude sensitivity measurements," J. Opt. Soc. Am. 51, 422-429 (1961).

26. P. G. J. Barten, Contrast Sensitivity of the Human Eye and its Effects on Image Quality (SPIE, 1999), Vol. 72.

27. S. Gibet and P.-F. Marteau, "Approximation of curvature and velocity for gesture segmentation and synthesis," in International Gesture Workshop (Springer, 2007), pp. 13-23.

28. L. B. Bagesteiro and R. L. Sainburg, "Handedness: dominant arm advantages in control of limb dynamics," J. Neurophysiol. 88, 2408-2421 (2002).

29. C. Hornsteiner and J. Detlefsen, "Characterisation of human gait using a continuous-wave radar at $24 \mathrm{GHz}$," Adv. Radio Sci. 6 , 67-70 (2008).

30. L. L. Long and M. Srinivasan, "Walking, running, and resting under time, distance, and average speed constraints: optimality of walkrun-rest mixtures," J. R. Soc. Interface 10, 20120980 (2013). 
31. H. Levitt, "Transformed up-down methods in psychoacoustics," J. Acoust. Soc. Am. 49, 467-477 (1971).

32. F. A. A. Kingdom and N. Prins, Psychophysics: A Practical Introduction (Academic, 2016).

33. J. L. Hintze and R. D. Nelson, "Violin plots: a box plot-density trace synergism," Amer. Statist. 52, 181-184 (1998).

34. J. C. Lagarias, J. A. Reeds, M. H. Wright, and P. E. Wright, "Convergence properties of the Nelder-Mead simplex method in low dimensions," SIAM J. Optim. 9, 112-147 (1998).

35. M. G. M. Stokkermans and I. E. J. Heynderickx, "Temporal dark adaptation to spatially complex backgrounds: effect of an additional light source," J. Opt. Soc. Am. A 31, 1485-1494 (2014).

36. T. N. Cornsweet, "The staircase-method in psychophysics," Am. J. Psychol. 75, 485-491 (1962).

37. R. M. Rose, D. Y. Teller, and P. Rendleman, "Statistical properties of staircase estimates," Atten. Percept. Psycho. 8, 199-204 (1970).
38. C. Kaernbach, "Adaptive threshold estimation with unforced-choice tasks," Atten. Percept. Psycho. 63, 1377-1388 (2001).

39. C. M. Colgan, "Critical flicker frequency, age, and intelligence," Am. J. Psychol. 67, 711-713 (1954).

40. N. Ginsburg, M. Jurenovskis, and J. Jamieson, "Sex differences in critical flicker frequency," Percept. Mot. Skills 54, 1079-1082 (1982).

41. M. Rea, The IESNA Lighting Handbook, 9th ed. (The Illuminating Engineering Society of North America, 2000).

42. ASSIST, Assist Recommends... Application Considerations Related to Stroboscopic Effects from Light Source Flicker (Lighting Research Centre, 2015).

43. M. Perz and D. Sekulovski, "Acceptability criteria for the stroboscopic effect visibility measure," in CIE 2017 Midterm Meeting "Smarter Lighting for Better Life," Jeju, South Korea (CIE, 2017). 